\title{
Calificación y trabajo en la industria automotriz
}

\author{
Jorge Carrillo V.*
}

En este artículo se analiza la estructura de las calificaciones de ios trabajadores en dos plantas automotrices en México, una tradicional enfocada al mercado interno, y otra moderna enfocada a la exportación, para contrastar, en forma empírica, el debate sobre la pérdida o no de la capacidad de decisión de ios trabajadores sobre su propio proceso de trabajo. Se parte del supuesto de que la automatización no implica necesariamente un proceso de descalificación; por el contrario, significa un cambio en las calificaciones y en su segmentación. Para corroborar la validez de las hipótesis planteadas se utiliza la herramienta estadística de los modelos loglineales. Entre las conclusiones, se pueden mencionar las siguientes: que las estructuras organizativas de las dos plantas difieren; que existen cambios en la política laboral de promoción en la empresa automatizada, basados en la capacitación del trabajador, y no en la antigüedad como en la planta tradicional (es decir, el criterio de eficiencia cobra gran relevancia). Estos cambios contribuyen a la centralización del control sobre los trabajadores, pero este control tiene poco que ver con la calificación per se, entendida como oficio. El trabajador no está perdiendo capacidad de decisión sobre la forma de trabajo con estas transformaciones, lo que está perdiendo, según señalan ios resultados de los modelos, es un momento en la negociación de las políticas de trabajo.

\section{Introducción}

La calificación en el trabajo en el sector fabril ha sido una preocupación importante desde mediados del siglo pasado. Un tema generalizado de discusión ha sido el de la transferencia de calificación del trabajador hacia la empresa. Autores como Ure señalaban desde 1845 que ésta debería consistir en poner al trabajador al simple ejercicio de la vigilancia y la destreza en la producción. Babbage, por su parte, proponía en 1832 sustituir obreros calificados por otros sin calificación, a través de la producción desde lugares donde resultara la más alta rentabilidad para las empresas. Desde entonces, la discusión sobre la calificación ha girado en torno al abaratamiento de la mano de obra y a la capacidad de decisión del trabajador sobre su propio proceso de producción.

Desde principios de la década presente, la discusión sobre la calificación recobra una gran importancia debido a que el creciente proceso de incorporación de nuevas tecnologías en los diferentes sectores industriales está modificando no sólo la estructura de las calificaciones dentro de

* Investigador de El Colegio de la Frontera Norte. El autor agradece los valiosos comentarios de Brígida García y la lectura con lupa y las valiosas sugerencias de Fernando Cortés. 
las empresas, sino la propia función que debe cumplir el trabajador en relación al proceso productivo.

Como señalan Auguren y Edgren (1983), a partir de los nuevos procesos tecnológicos se proclaman conceptos de nuevas fábricas y la eliminación de la cadena de producción. Los estadunidenses Piore y Sabel (1984) consideran que la producción industrial lleva a la formación de una especialización flexible. Kern y Schumann (1984) pronostican un cambio paradigmático en la política laboral, consistente en el abandono del taylorismo por nuevos conceptos de producción con funciones integrales y mayores requisistos de calificación. Dhose, Jüergens y Malsch (1986) mencionan la nueva etapa tecnológica-organizativa como la de mayor control sobre el trabajo basada en la autorregulación central cercana a la producción.

Las implicaciones de la incorporación de modernas tecnologías en el proceso técnico y en la administración del trabajo ha sido interpretada, grosso modo, de dos maneras: mientras que unos hablan de lagunas ampliadas del control o de espacios laborales flexibles, o incluso del fin de la división taylorista del trabajo (Kern y Schumann, 1984), otros pronostican un control intensificado en el tiempo de trabajo sobre el personal y una intensificación del mismo (Coriat, 1981). En otros términos, a la mayor automatización corresponde un proceso de evolución de ciertos trabajos calificados, la inserción de nuevas especialidades y un cierto margen de desarrollo sobre la producción por los propios trabajadores (Burawoy, 1983; Thompson, 1983; Malsh, 1984; Womack, 1986), o por el contrario se presenta un proceso de descalificación, degradación en el trabajo, y sobre todo, un mayor control sobre los trabajadores (Bright, 1958, Braverman, 1974; Coriat, 1981; Shaiken, 1986).

La relevancia de estas discusiones para México y en especial para el norte del país es significativa por tres razones: en primer término, existe un apogeo del sector; decenas de plantas se han establecido en esta zona con el fin de producir y exportar hacia Estados Unidos; cerca de 140 plantas de ensamble y de autopartes, se encontraban operando en diez ciudades en dicha zona en 1987 (véase cuadro 1) y mantienen el segundo lugar nacional en la generación de divisas. En segundo término, porque el proceso de centralización de la producción en unas cuantas empresas automotrices se ha exacerbado. Desde 1979, cuando General Motors se estableció en la frontera, han continuado instalándose las distintas transnacionales del auto. General Motors, Ford y Chrysler ocupaban en 1987 a cerca de 38 mil empleados (más de $60 \%$ del total ocupado en esta zona) (véase cuadro 1-A). $\mathrm{Y}$, en tercer término, porque dichas plantas han incorporado, en forma más intensiva, procesos de innovación tecnológica. Una visita a nueve plantas estadunidenses que realicé en ese año, me permite asegurar que existe un proceso de difusión de tecnología dura y 
blanda en las plantas de ensamble para la exportación ${ }^{1}$ a través de la incorporación de procesos técnicos automatizados, y la flexibilidad en sus sistemas de administración del trabajo como el justo a tiempo, los equipos de trabajo y los círculos de calidad. ${ }^{2}$

Otro aspecto importante al estudiar la calificación en la industria automotriz es que el desarrollo de plantas de alta tecnología y de sistemas flexibles de administración ha roto con el tradicional ensamblaje basado en

CUADRO 1

Empleo en plantas de ensamble automotriz para la exportación. Norte de México (junio de 1987)

\begin{tabular}{lcccc}
\hline \multirow{2}{*}{$\begin{array}{l}\text { Zona } \\
\text { norte }\end{array}$} & Maquilas & No maquilas & Empleo $^{*}$ & $\begin{array}{r}\text { Promedio de } \\
\text { trabajadores } \\
\text { por planta * * }\end{array}$ \\
\cline { 2 - 3 } $\begin{array}{c}\text { Municipios } \\
\text { fronterizos }\end{array}$ & 95 & 17 & 48912 & 562.2 \\
$\begin{array}{c}\text { MunIcipios } \\
\text { no fronterizos }\end{array}$ & 20 & 7 & 8803 & 1257.5 \\
$\begin{array}{c}\text { Total } \\
\text { notablecimientos }\end{array}$ & 115 & 24 & 57715 & \\
\hline
\end{tabular}

Fuente: Elaboración del autor con base en el Directorio de Industrias Maquiladoras de Secofi, junio de 1987, y en visitas a plantas.

* En $32.4 \%$ de los establecimientos no se pudo verificar el empleo.

** Con base en las 94 plantas que reportaron empleo.

1 La incorporación de tecnología en la industria automotriz en México es analizada, para algunos casos, en Arteaga y Carrillo (1988).

2 El sistema justo a tiempo esta diseñado para disminuir el tiempo de producción y controlar las incertidumbres productivas. Es una de las técnicas más importantes que los japoneses han utilizado para mejorar la calidad e incrementar la productividad en los procesos de manufactura, produciendo el mínImo necesario de unidades, en cantidades menores y en el menor tiempo posible. AsimIsmo considera la eliminación de inventarios y desperdicios.

Los equipos de trabajo son una forma de organIzación del trabajo en forma colectiva; están formados de acuerdo con la tecnología, los procesos de integración secuencial y el área geográfica. Los equipos se conforman de 8 a 12 trabajadores regularmente, y se establecen por requerimiento de personal del área, hacen actividades semejantes y tienen un facilitador o coordinador. Este es un trabajador perteneciente al equipo, que coordina y apoya las actividades, el cual es rotado permanentemente entre los miembros del mismo. Dentro de cada grupo existe un seguimiento permanente de cada trabajador para evaduar su entrenamiento, habilidad y versatilidad. Con los equipos de trabajo se busca mayor integración de los trabajadores con los objetivos de la empresa y mayor responsabilidad, así como que cada trabajador conozca perfectamente todas las actividades de su equipo. Los círculos de calidad son juntas que realizan los trabajadores y el supervisor para detectar problemas de calidad, analizar los motivos de errores y proponer soluciones. Se reúnen diariamente todos los miembros de la línea o de un área determinada con su supervisor, y semanalmente se reúnen con superintendentes y coordinadores generales de los círculos. Al igual que en los equipos se busca el involucramiento de los trabajadores con el trabajo y una mayor responsabilidad. 
CUADRO 1-A

Empleo en empresas automotrices estadunidenses.

Orientación exportadora zona norte (junio de 1987)

\begin{tabular}{|c|c|c|c|c|}
\hline $\begin{array}{l}\text { Multina- } \\
\text { cionales }\end{array}$ & $\begin{array}{c}\text { Estable- } \\
\text { cimientos }\end{array}$ & Empleo & $\begin{array}{l}\text { Promedio de } \\
\text { trabajadores } \\
\text { por planta }\end{array}$ & $\begin{array}{c}\text { Principales } \\
\text { productos }\end{array}$ \\
\hline \multicolumn{5}{|l|}{ Frontera } \\
\hline Ford & 8 & 6587 & 2195.7 & $\begin{array}{l}\text { Vestiduras } \\
\text { Radiadores }\end{array}$ \\
\hline G. M. & 11 & 20931 & 1902.8 & Arneses \\
\hline Chrysler & 2 & 2727 & 1363.5 & Arneses \\
\hline \multicolumn{5}{|l|}{ No frontera } \\
\hline Ford & 3 & 1718 & 859.0 & $\begin{array}{l}\text { Ensam. de } \\
\text { aut/motores }\end{array}$ \\
\hline G. M. & 5 & 3200 & 3200.0 & $\begin{array}{l}\text { Ensam. de } \\
\text { aut } / \text { motores }\end{array}$ \\
\hline Chrysler & 1 & 2000 & 2000.0 & Motores \\
\hline
\end{tabular}

Fuente: la misma del cuadro 1.

* El promedio de trabajadores por planta fue construido exclusivamente entre las plantas que reportaron número de empleados.

el uso de fuerza intensiva de trabajo no calificado en las maquiladoras para la exportación. A partir de los postulados de la teoría de la nueva división internacional del trabajo se esperaba que México se especializara en operaciones intensivas en mano de obra y se pronosticaba un gran riesgo en la existencia de las plantas en la medida en que se fueran automatizando. El desplazamiento de mano de obra por las máquinas automáticas y el requerimento de mano de obra altamente calificada implicaría por tanto el traslado de plantas del norte de México hacia Estados Unidos. Al inicio de la década, una firma norteamericana de consultoría llegó incluso a señalar un descenso industrial paulatino en la maquiladora, a partir de los procesos de automatización que vivían las compañías matrices en Estados Unidos, y hablaba de que tomando como base 100 los establecimientos en 1981, la reducción alcanzaría $39 \%$ para 1989 . Difícilmente resultará válido tal pronóstico, toda vez que el empleo y los establecimientos de la maquila han crecido en forma rápida, tanto los intensivos en trabajo, como los intensivos en capital con procesos automatizados.

La cuestión general sobre la incorporación de tecnología y formas flexibles de administración del trabajo y su relación con la calificación y con la capacidad de decisión de los trabajadores sobre su propio proceso de trabajo, pueden abrir diversas líneas de investigación.

Por ejemplo, relacionadas con el propio concepto de calificación, la estructura de calificaciones y la función de las mismas; con el impacto 
en el empleo y la función de los sindicatos en este proceso; con las estructuras de control empresariales y las formas de resistencia en el trabajo; con el cambio en el perfil socioeconómico de los trabajadores y su percepción y actitud hacia estos nuevos conceptos y formas de producción, etc. Aquí me preocupo sólo por una de las implicaciones de la tecnología en la calificación: la función de ésta en las políticas de movilidad ascendente. La política de movilidad dentro de los mercados internos de trabajo es un proceso escasamente estudiado y que resume parte de esta discusión sobre control de la empresa en el trabajo y, por ende, está relacionado con la capacidad de decisión de los trabajadores sobre su propio proceso de trabajo.

Habiendo señalado lo anterior, me resta presentar en esta introducción el objetivo principal del trabajo: analizar la estructura de las calificaciones en dos plantas automotrices en México, una tradicional y otra moderna, a la luz del debate sobre la pérdida o no de la capacidad de decisión de los trabajadores sobre su propio proceso de trabajo. ${ }^{3}$ La idea central es demostrar empíricamente que en la empresa orientada hacia el mercado interno y ubicada en el centro del país, la antigüedad es la variable que explica la calificación y la movilidad en el trabajo. Mientras que en la empresa orientada hacia la exportación y establecida en el norte, la calificación es la variable que explica la movilidad. Los supuestos que están detrás de estos objetivos son que el fenómeno de la automatización no viene acompañado de un proceso de descalificación sino de un cambio en las calificaciones y en su segmentación, y que la capacidad de decisión del obrero sobre su trabajo se incrementa a la par que el control de la empresa sobre él.

\section{El debate político sobre la tecnología y la calificación}

Definir el concepto de calificación reviste un primer problema de disciplina. Mientras que para algunos economistas y demógrafos la calificación representa un problema de medición, el cual normalmente es resuelto distinguiendo la posición que ocupan las personas dentro del trabajo (clasificados con referencia a la propiedad o a la posición de trabajo), para algunos sociólogos significa básicamente un problema de conceptuación, en la medida que permite explicar los espacios donde se expresan las relaciones entre el capital y el trabajo.

El examen de las obras que presento atiende a un problema central: la mayor o menor calificación, derivada de la automatización y de los modelos flexibles de organización, ¿basta por sí misma para definir si están perdiendo los trabajadores capacidad de decisión en el trabajo? La respuesta

3 Esta capacidad está referida a la forma de trabajo, a la intensidad en el mismo y al control sobre los tiempos muertos, fundamentalmente. 
anticipada es negativa y se relaciona con la hipótesis central de este trabajo: la calificación, en las industrias desarrolladas bajo la política industrial de sustitución de importaciones, pasó de ser un momento de la negociación colectiva, a través de la antigüedad, a ser un momento del control empresarial en las industrias orientadas a la exportación. En otras palabras, la pérdida en la capacidad de decisión por parte de los trabajadores no recae en su trabajo mismo o en la forma como éste es ejecutado, sino más bien en las condiciones bajo las cuales se desarrolla ese trabajo. De ahí que el análisis de las políticas de movilidad en el empleo, que serán analizadas en dos plantas, sean centrales para comprender esta pérdida de espacios de control.

La sociología ha hecho importantes aportaciones acerca de las implicaciones de la modernización tecnológica y los cambios en la calificación. Especial interés han tenido las implicaciones que, a partir de cambios en el proceso técnico, afectan al poder de los trabajadores sobre su propio proceso de trabajo. La calificación tiene aquí claramente una connotación política.

Ya desde 1958, autores como Bright y Diebolt observaron la importancia de relacionar los avances tecnológicos en la empresa con la calificación y los puestos de trabajo. Brigth establecía una curva para ejemplificar cómo a mayor complejidad tecnológica (automatización) descendían los requerimientos de calificación del puesto de trabajo (ergo del trabajador). Diebolt (1958) observaba algo similar.

Con la amplia difusión de la tecnología desde los años setenta, se generaliza esta posición. Varios autores encuentran que, con el desarrollo de la tecnología, se presenta, por un lado, una disminución de la capacidad de decisión de los trabajadores sobre su propia actividad y una pérdida de poder político de la mano de obra, tanto individual como organizativamente; y por otro lado, un deterioro de la situación económica, laboral y psíquica de los trabajadores (Lettieri, 1972; Braverman, 1974; Coriat, 1981; De la Garza, 1984, y Shaiken, 1986, entre otros). Howard (1982) senala, por su parte, que la creación de nuevos puestos en empresas automatizadas no está dirigida a los trabajadores sindicalizados.

Otros autores como Thompson (1983) y Littler (1981), critican las tesis anteriores, especialmente la de Braverman. Consideran que la automatización no provoca un proceso de descalificación y degradación del trabajo pues existe todavía un considerable grado de control de los trabajadores calificados, y en general, de los obreros, sobre el proceso de producción; y, en la política sindical, la calificación es más bien un momento salarial del contrato, que un problema de aprendizaje real. Esta posición interpreta los cambios hacia la flexibilidad en las nuevas formas de organización como una transformación en el verticalismo autoritario sobre bases de una mayor equidad entre trabajadores de diferentes jerarquías (Malsch et al., 1984 y Womack, 1986). 
Una posición intermedia es la de Burawoy (1983), quien interpreta estos cambios como una "invasión de la empresa sobre el espacio del trabajo", fundamentada sobre bases tradicionales pero con movilización del consenso para incrementar la productividad. Burawoy encuentra, por un lado, una resistencia obrera generalizada que contrarresta los efectos del cambio tecnológico; y por otro lado, que los trabajadores mismos tienden a autocontrolarse y a aceptar reglas, por lo que no siempre es necesario un cambio coercitivo para que sea eficiente la estructura de control. El control consensual, en este sentido, puede jugar un papel más importante dentro de las nuevas calificaciones.

Esta discusión está directamente relacionada con el propio concepto de organización y la definición del papel que deben jugar la calificación y el trabajador dentro de la empresa en los modelos organizativos. En el modelo fordista, con origen en Taylor y sus antecesores, se considera que hay que despojar de todo el conocimiento técnico al trabajador, debido a que éste hace un mal uso de sus conocimientos en bien de sí mismo y en contra de la empresa, que hay que fragmentar el trabajo lo más posible para incrementar la productividad del trabajador y que hay que incentivarlo económicamente para que produzca más. Se parte de que el trabajador, que detenta un mayor control de su actividad, es más ineficiente, más conflictivo y, en fin, incapaz de poner sus conocimientos al servicio de la organización empresarial. Por tanto, el supuesto central en las empresas automotrices diseñadas bajo el fordismo es hay que "des-calificar" al trabajador y al trabajo mismo.

Mientras que el modelo flexible (como el modelo japonés ${ }^{4}$ también conocido como toyotismo), conceptúa al trabajador como un agente productivo, creativo e innovador, capaz de integrarse a los intereses de la empresa, motivo por el cual se delegan en él responsabilidad y decisiones. Este modelo, que acompaña a las nuevas tecnologías, ha implicado una mayor flexibilidad en el trabajo (intercambio de obreros en turnos, actividades y departamentos), una menor rigidez en las jerarquías (tienden a desaparecer diferencias entre obreros de producción, de mantenimiento, de control de calidad y supervisores) y una mayor responsabilidad. Los círculos de calidad, los grupos o equipos de trabajo y la permanencia constante en el empleo, son tres factores clave para el éxito de este modelo. El supuesto central, entonces, en empresas automotrices automatizadas organizadas bajo el modelo japonés, es que hay que re-calificar el trabajo.

El debate iniciado desde los años sesenta sobre el proceso de descalificación o de recalificación a partir de la tecnología y sus implicaciones en el control, se fortalece en los ochenta. La discusión actualmente no radica en que si con tecnología hay más o menos calificación en el trabajo,

\footnotetext{
${ }_{4}^{4}$ Para una mayor discusión sobre el modelo japonés véase Moritani [1985].
} 
pues aun los críticos de la modernización tecnológica han reconocido que en esta fase se incrementa la ocupación de mano de obra calificada, las responsabilidades y la flexibilidad, ${ }^{5}$ sino en que si a esas nuevas calificaciones o recalificaciones en el trabajo, corresponde o no un mayor control, o quizás un tipo de control distinto.

Recientemente, Dhose, Jurgens y Malsch avanzan en esta discusión al señalar que, más que operar los trabajadores en las fábricas automatizadas sobre un producto individual laboran sobre las perturbaciones en las instalaciones, con lo cual la función de la calificación es desligada del producto, separada de la repetitividad y, por tanto, se vuelve menos determinista y más contingente. De este modo, el propio trabajo llega a ser menos controlable. Estos autores mencionan que casi todas las tesis de autonomía que se refieren a los trabajadores automatizados, derivan de la tesis de contingencia. No se trata -mencionan- de una alternativa al fordismo clásico sino de una sustitución de éste:

Así, podemos observar un desarrollo en dos polos: por un lado, se está perfilando un modo de regulación que está encaminado a una mayor descentralización de la responsabilidad de producción, a una mayor integración social y una mayor autonomía de trabajo por parte del personal. Pero, por el otro lado, observamos un nuevo énfasis en el control central, en la dirección y evaluación de tales procesos de innovación, así como en diversos intentos para desarrollar nuevas estructuras de control. No se puede hablar por lo tanto de una sustitución sin condiciones del fordismo por un modo de regulación nuevo claramente delineado (Dhose, Jurgens y Malsch, 1986).

Dentro de estas "nuevas condiciones" los equipos de trabajo cumplen una función central en los cambios de la estructura organizativa. ${ }^{6}$ Además, forman parte primordial de los conceptos de dirección y control. Los modelos de equipo son una forma de trabajo en grupo no estática; los trabajadores individuales reciben sus propias instrucciones y además, cada equipo tiene asignado un complejo de tareas. Tales equipos, en su forma más desarrollada, organizan autónomamente la división del trabajo dentro del grupo, se ocupan de la rotación en el empleo y seleccionan a un coordinador (facilitador) como director del equipo. Nuevas formas de remuneración basadas en bonos económicos y en el escalafón basado en la polivalencia (habilidad y conocimiento para desarrollar más operaciones), motivan la rotación en el empleo, así como el aumento en la flexibilidad en el mismo. Los equipos de trabajo funcionan en ciclos

\footnotetext{
5 Touraine (1963), Naville (1963) y Di Telia (1971). Otros autores encuentran que, además, se presenta una segmentación en la estructura de las calificaciones y una creciente ocupación de técnicos e ingenieros. Malsch (et al.,1984), Dohse et al. (1984) y Womack (1986).

${ }^{6}$ El trabajo colectivo en equipo y la función.que debe cumplir, no es un tema nuevo para la sociología. Con Durkheim se planteó, por vez primera, la función de la cohesión al interior
} 
cortos, conformados entre 8 y 12 trabajadores, y no son exclusivos del trabajo automatizado. En la mayoría de las empresas maquiladoras norteamericanas de autopartes, intensivas en trabajo, como las de ensamble de vestiduras o de arneses, los equipos de trabajo son la forma central del cambio en la organización del trabajo. Igual importancia tienen los equipos en las ensambladoras de automóviles en Hermosillo, Chihuahua o Saltillo.

Finalmente, la discusión sobre calificación y su impacto en las estructuras de control en empresas automotrices implica la investigación en diversos planos tanto analíticos como de la realidad. En este trabajo, por problemas de espacio, sólo tomaré uno: el control medido a través de las políticas de movilidad.

Las políticas de movilidad han sido tradicionalmente un espacio de negociación entre las empresas y los sindicatos. La capacidad de decisión de quién y bajo qué circunstancias ascienden o no los trabajadores, está relacionada con las carreras profesionales dentro de las empresas y con el poder que sustentan tanto la gerencia como el sindicato. La movilidad se refleja, básicamente, en dos aspectos, el salario y el puesto de trabajo. Y estos dos aspectos son determinados, en gran parte, por la política de calificación. De ahí que el vínculo entre estructura de calificación y la política de movilidad permite analizar la tecnología y las estructuras de control empresarial. Veamos dos casos de plantas automotrices.

\section{Movilidad y calificación en empresas tradicionales y modernas}

Para contrastar los cambios en la calificación y su impacto en las estructuras de control y en la movilidad salarial en empresas de tecnología rígida y de tecnología flexible, he seleccionado dos plantas automotrices pertenecientes a la misma firma. Llamaré a la planta establecida en el área metropolitana de la ciudad de México "planta ISI", y a la establecida en una ciudad importante del norte del país "planta Export"' ? En cada caso, empezaré con una breve descripción del proceso de trabajo que permita contextualizar los resultados de los datos recabados. La información proviene de las nóminas de trabajadores sindicalizados realizadas por las propias empresas en 1986. Se trata, por tanto, en los dos casos, de censos.

\footnotetext{
de las estructuras fabriles (Durkheim, 1893). Naville (1963) y Diebold (1958) encuentran una relación entre los equípos de trabajo y la optimización del uso de nuevas tecnologías. Naville (1963) y Touraine (1963) coinciden en que los equipos de trabajo y los cambios en la división del trabajo son producto de la automatización y cumplen una función primordial. Malsch et al. (1984), Dhose et al. (1984) y Womack (1986) trabajan sobre esta misma línea.

7 La llamo planta ISI porque surge bajo la política de Industrialización por sustitución de importaciones, y a la planta Export porque se establece bajo la polítlca gubernamental de industrialización para la exportación.
} 
Defino en este trabajo la calificación como una situación de evolución profesional (véase Touraine, 1963), es decir, como expresión de una relación social de producción en donde diferentes indicadores, que se sintetizan en los trabajadores, son ejercidos en una situación laboral ante puestos de trabajo con requerimientos específicos. Por tanto, la evolución profesional se da en un marco donde se relacionan atributos del trabajador, situación en la que se ejerce la actividad y atributos del puesto de trabajo.

Se revisaron para el análisis las siguientes variables base: identificación del trabajador, puesto ocupado, departamento de producción, planta en la que labora, año en que entró a la planta, salario y compensación por hora. Se construyeron tres variables dicotómicas y una tricotómica para medir la importancia de la calificación: 1) Puesto. Es el puesto de trabajo en donde se distingue, con base en la función del mismo, entre puestos directos (OD) (operadores de ensamble más soldadores, operadores en manejo de materiales, carroceros y operadores de moldeo) y aquellos de mantenimiento y control de calidad (OM) (también incluye obreros de prueba de afilado, mecánicos, especialistas e inspectores). 2) Antigüedad. Es el año en que entró por vez primera a la empresa, distinguiendo entre baja (BA) y alta (AA) (ponderada diferencialmente para cada planta). 3) Calificación. Es la categoría de calificación otorgada al puesto de trabajo según las evaluaciones de puesto de la propia empresa; ocho categorías de calificación fueron clasificadas en baja (BC) y alta (AC). 4) Movilidad. Diferencia del salario que corresponde a la categoría de calificación del puesto de trabajo y el que nominalmente recibe el obrero. Esta última variable tiene los siguientes rangos: movilidad salarial ascendente (MSA) si el trabajador gana más de lo que le corresponde por calificación al puesto que ocupa, sin movilidad (SM) si gana lo mismo, y con movilidad salarial descendente (MSD) si percibe menos.

\section{Planta ISI}

La planta ISI fue concebida a principios de los años sesenta bajo la política de sustitución de importaciones. En realidad más que una planta se trata de un complejo donde varias plantas se encuentran integradas por una sola administración. Produce varios modelos de autos para el mercado nacional y trabaja con el modelo de organización fordista. La calificación en esta planta tradicional estaría - tomando como base la definición anterior- en el siguiente estado de evolución:

Producción en serie. La mayoría de las operaciones ocupadas por obreros especializados son limitadas, rápidamente aprendidas y repetitivas. Los trabajadores calificados se ubican en los talleres de mantenimiento y reparación. Predominio de la organización centralizada del trabajo sobre el trabajador directo. La habilidad —como experiencia- no es ya el principio central del funcionamiento de la fábrica, aunque el trabajo no puede realizarse sin recurrir al obrero calificado. Esta fase está ligada a la previ- 
sibilidad técnica (exigencia de normas y productos elaborados) y económica (programas de fabricación a largo plazo con voluminosos inventarios). La producción en serie se basa en procesos técnico-profesionales y técnico-económicos. Los puestos de trabajo definen al obrero especializado y la política para ascender a los puestos más calificados está basada en la antigüedad (como se tratará de demostrar). Coinciden y se oponen el trabajador de oficio (en mantenimiento y maquinaria) y el trabajador especializado (en la cadena de montaje fordista).

La producción se inicia con la fundición del fierro para producir monoblocks. La actividad desempeñada en esta área es difícil, ya que se trabaja con altas temperaturas en los hornos y en el recorrido de los moldes. Las máquinas-herramientas que separan el monoblock del molde producen un ruido que es similar en decibeles al de los aviones cuando despegan. Aunque todos los trabajadores cuentan con equipo de protección, éste no es suficiente. Se trata de trabajadores especializados que tienen más de 10 años de antigüedad y que operan con máquinas-herramientas de más de 20 años de uso. Por ser ésta la planta más tradicional del complejo y con altos costos, la empresa ha decidido modernizarla e incrementar la productividad y la calidad.

Para ello a mediados de 1985 se estableció el pacc (Participación Activa para la Calidad y Competitividad). Los objetivos que buscaba la empresa a través del PACC fueron los siguientes: 1) lograr calidad de exportación; 2) lograr competitividad de exportación, y 3) conservar la planta de fundición. Para lograr esto se ha buscado: a) que se respeten con puntualidad los horarios de trabajo (de 6 a.m. a 3 p.m.); b) eliminar los tiempos muertos (por ejemplo que se deje de tomar café en la mañana, un receso que normalmente duraba 20 minutos); c) incrementar las coladas (en la planta de fundición) de 420 a 600; d) mejorar los servicios y cursos de capacitación, y e) implantar una nueva fuerza de trabajo que tienda a incrementar la productividad y la habilidad.

La idea global es que, iniciando con la planta "menos eficiente" como la de fundición, y siguiendo con la de motores, se lograría implantar un sistema organizativo diferente que incrementara la productividad y calidad en el trabajo a través del establecimiento de un modelo más flexible basado en una mayor participación de los trabajadores y del sindicato. El PACC se puso en marcha en 1985, y hasta mediados de 1987 no había logrado terminar con los tiempos muertos no sólo por enfrentarse a viejas prácticas y costumbres de los trabajadores sino porque los trabajadores tienen un sindicato oficial, fuerte y democrático. ${ }^{8}$

En lo que respecta a la planta de motores se realiza, primero, el torneado y pulido del monoblock con grandes máquinas electromecánicas,

${ }^{8}$ La clasificación es tomada del estudio de Roxborough (1987). 
y posteriormente el ensamble de sus partes. Se opera sobre el producto en la tradicional cadena fordista y las cuotas de producción están fijadas por línea y negociadas en parte con el sindicato.

Los tiempos muertos son también aquí parte de la tradición, y están siendo reducidos a través del PACC. Para llevar adelante este programa se formaron en la planta de ensamble los grupos participativos voluntarios. Un elemento importante es que los trabajadores se resistieron a la implantación de este proyecto de restructuración organizacional dado que las ventajas "eran sólo para la empresa" y se terminaba "con costumbres de 20 años". Ello dio lugar a que varios miembros del comité central del sindicato fueran reticentes hacia el PACC.

La maquinaria es menos antigua en esta planta que en la de fundición y algunos procesos están computarizados. En la escala de niveles de mecanización de Brigth, la planta quedaría clasificada como mecánica o de baja automatización (Bright, 1958).

Finalmente, en la planta de ensamblado general, las carrocerías son punteadas en gran parte con dos robots para posteriormente pasar por un sistema moderno de pintura. A lo largo de la cadena son ensambladas todas las autopartes. En la escala Brigth se trataría de un nivel donde la máquinas han sido puestas bajo control externo; una planta semiautomatizada. Una muestra de autos pasa diariamente por un examen exhaustivo de calidad. La cadencia fordista en esta planta es ocupada con trabajadores especializados organizados, nuevamente, en una gama de puestos claramente definidos. En los espacios de trabajo está restringida la actividad de los supervisores por los delegados departamentales que, aunque dependientes de una estructura sindical muy jerarquizada, funcionan como intermediarios en los problemas cotidianos entre los mandos medios de control de la gerencia y los trabajadores de piso.

La capacidad de negociación sindical juega un papel central en el complejo industrial, al igual que en las otras plantas automotrices orientadas al mercado interno, y contaba hasta mediados de 1987 con uno de los mejores contratos colectivos del ramo. La media de antigüedad de los trabajadores en la planta es de 12 años y la mayoría tiene más de 34 años de edad. Observemos a continuación los resultados del análisis.

La estructura organizativa es compleja y muy definida: existen 46 departamentos de producción distribuidos en cuatro plantas y están definidos 240 puestos de trabajo (en 1985 eran 303 puestos). El padrón de trabajadores registró 3761 personas en noviembre de 1986, y la distribución en las variables construidas fue la siguiente: 


\section{CUADRO 3}

Características de la planta ISI

\begin{tabular}{lrr}
\hline \multicolumn{1}{c}{ Variables } & Frecuencias & Porcentaje \\
\hline $\begin{array}{l}\text { Puesto } \\
\text { Obreros directos }\end{array}$ & 2000 & \\
Obreros de mantenimiento & & 53.2 \\
y control de calidad & 1003 & 26.6 \\
Resto & 758 & 20.2 \\
Antigüedad & & \\
Baja (0-7 años) & 1321 & 35.3 \\
Alta (8-41 años) & 2434 & 64.7 \\
Calificación & & \\
Baja & 1826 & 48.7 \\
Alta & 1926 & 51.3 \\
Movilidad salarial & & \\
Descendente & 191 & 5.0 \\
Sin movilidad & 2206 & 58.7 \\
Ascendente & 1364 & 36.3 \\
\hline
\end{tabular}

Se puede apreciar que la mayoría de los trabajadores ocupan puestos directos de producción (obreros especializados en la cadena fordista) y sólo una parte puestos de control y mantenimiento. La nada despreciable cantidad de $20 \%$ pertenece a las familias de aseadores, cafetería, carpinteros, oficina, vigilancia y choferes. En cuanto a la antigüedad, la mayor parte de los trabajadores tienen entre 8 y 41 años laborando en este complejo y, según líderes sindicales entrevistados, en general tienen experiencia laboral anterior. Sólo $35 \%$ tenía entre 0 y 7 años trabajando aquí. La media de antigüedad resultó de 13 años. La calificación - como atributo del puesto de trabajo- resultó ser alta para $51.3 \%$ y baja para $48.7 \%$.

Para observar el comportamiento de las variables se seleccionó el modelo de análisis loglineal. ${ }^{9}$ La hipótesis de trabajo para la planta orientada hacia el mercado interno es la siguiente: en el estado de evolución profesional del trabajador especializado, la antigüedad es un factor que se asocia con el lugar de trabajo que se ocupe, y por ende, con la movilidad salarial y con la calificación. La proposición teórica sería que en plantas automotrices tradicionales.eon organización fordista en el trabajo y orientadas al mercado interno, la mayor antigüedad está relacionada con la calificación, con el puesto de trabajo y con la movilidad económica.

9 La principal razón es que permite trabajar con variables cualitativas y contrasta supuestos teóricos generando modelos de interacción de las variables (véase, García, 1987). 
La conexión de la hipótesis con la teoría es que, en la fase previa a la automatización, los trabajadores son especializados y, dado que existe una estructura organizativa rígida y una estructura de control mediada por la capacidad de negociación sindical, el escalafón ciego es el patróñ que determina la movilidad. Esto es, el ascenso en la categoría de calificación, en el puesto que se ocupa, y en la movilidad económica está determinado por la antigüedad en la empresa. Una mayor capacidad de decisión del operario de la forma como se ejecuta el trabajo estaría acompañada de una política de ascenso de este tipo. ${ }^{10}$

De acuerdo con la hipótesis, el modelo deberá tomar en cuenta las interacciones entre antigüedad, calificación, puesto y movilidad. El modelo diseñado originalmente fue el siguiente: la antigüedad define el puesto que se ocupa, y con ello, la calificación asignada. Y la movilidad económica estaría dada por la antigüedad y consecuentemente por el puesto que se ocupa.

Se corrieron varios modelos y finalmente se decidió seleccionar el "modelo final generado", que muestra las interacciones que mejor se ajustan a los datos. El resultado fue el siguiente: existe una interacción de tres variables (antigüedad, calificación y movilidad) y tres interacciones (de las anteriores variables) de dos variables, todas ellas relacionadas con el puesto. El modelo se expresa así:

$$
\begin{aligned}
& \text { Antigüedad * Calificación * Movilidad } \\
& \text { Calificación * Puesto } \\
& \text { Antigüedad * Puesto } \\
& \text { Movilidad * Puesto }
\end{aligned}
$$

$\mathrm{Al}$ analizar los coeficientes lambda que se presentan en el cuadro 4 , donde se muestra la única interacción significativa de tres variables, resultó que hay una doble política económica en el empleo. A los trabajadores que cuentan con calificación alta desde que entran a la planta se les aplica una política de "escalafón ciego"; esto es, entran con un salario por debajo del que les corresponde al puesto de trabajo, y conforme avan-

10 Esta hipótesis se conecta con una más general sobre las diferencias en las estructuras de control en los modos de industrialización por sustitución de importaciones y en el modo de industrialización para la exportación. Presento a continuación la parte de la hipótesis que tiene relevancia con el trabajo que aquí se presenta: el bajo nivel de restructuración y el tipo de sindicalismo con mayor capacidad de negociación, en la fase de sustitución de importaciones, determina que se presente: a) un proceso de organización menos burocratizado, b) un tipo de autoridad representativa con base en la estructura jerárquica y c) una mayor capacidad de decisión de los trabajadores sobre el proceso de trabajo. En contraposición, el nivel alto de restructuración y el tipo de sindicalismo con menor capacidad de negociación en la fase de exportación, determina que se presente: a) una mayor burocratización, d) un tipo de autoridad mas flexible con base en el profesionalismo en el trabajo y c) una menor capacidad de negociación de los trabajadores sobre su proceso de trabajo. 
zan en la antigüedad les pagan más, hasta alcanzar el salario que les corresponde. Mientras que a los trabajadores que inician con baja calificación les pagan lo que corresponde a su puesto y conforme avanzan en antigüedad empiezan a tener una movilidad descendente. Es decir hay una política de relativa desvalorización de la fuerza de trabajo, inversa al "escalafón ciego", en donde los trabajadores que no logran avanzar en el escalafón dentro de la carrera profesional de la planta tienden a descender en la movilidad económica.

CUADRO 4

Interacción de antigüedad, calificación y movilidad salarial.

Coeficientes lambda; análisis loglineal

\begin{tabular}{lrrrrr}
\hline & \multicolumn{4}{c}{ Antigüedad } \\
\cline { 2 - 4 } \cline { 5 - 6 } Movilidad & Calif. baja & Calif. alta & Calif. baja & Calif. alta \\
\cline { 2 - 5 } Descendente & 0.40 & -0.40 & & -0.40 & 0.40 \\
Sin movilidad & -0.45 & 0.45 & & 0.45 & -0.45 \\
Ascendente & 0.05 & -0.05 & & -0.05 & 0.05 \\
\hline
\end{tabular}

Por lo que respecta a las tres interacciones de dos variables que resultaron del "modelo final generado", se refieren a la importancia del puesto de trabajo y su relación con la antigüedad, con la calificación y con la movilidad económica. En los cuadros 5, 6 y 7 se observan las lambdas de dichas interacciones.

Los resultados reflejan que existe una política de carrera profesional en la planta ISI, donde los obreros directos tienen baja antigüedad, por un lado, y tienen baja calificación, por el otro. Además no tienen movilidad -o están con el salario que les corresponde al puesto de trabajo. Conforme transcurren los años en la planta, avanzan en la carrera profesional de la propía empresa. De tal suerte que, al ser obreros de mantenimiento, tienen alta antigüedad, alta calificación y una movilidad que va en dos sentidos: descendente y ascendente. Relacionando el cuadro 4 con la búsqueda de explicación de esta doble política económica se puede desprender que es descendente si los obreros de mantenimiento entran desde un principio con alta calificación a trabajar. Y es ascendente si han alcanzado ser obreros de_mantenimiento a partir de la política de escalafón con base en la antigüedad.

Por tanto, podemos señalar lo siguiente como conclusión: en la planta ISI hay una política de "escalafón ciego" basada en la antigüedad. Se observa, además, una política de carreras profesionales. La antigüedad es una variable central en el movimiento dentro de la planta, pero esta varia- 
ble interactúa con la movilidad económica en forma diferencial para trabajadores que entran con o sin calificación. La política es de "escalafón ciego" y movilidad ascendente para los altamente calificados, y de "desvalorización" para los que no lograron mejorar su baja calificación. La antigüedad determina la movilidad económica según la calificación con que se entre a laborar.

CUADRO 5

Lambdas. Planta ISI

\begin{tabular}{lcc}
\hline Antigüedad & Obreros directos & Obreros mantenimiento \\
\hline Alta & -0.35 & 0.35 \\
Baja & 0.35 & -0.35 \\
\hline
\end{tabular}

CUADRO 6

Lambdas. Planta ISI

\begin{tabular}{lcc}
\hline & \multicolumn{2}{c}{ Puesto } \\
\cline { 2 - 3 } Calificación & Obreros directos & Obreros mantenimiento \\
\hline Baja & 1.14 & -1.14 \\
Alta & -1.14 & 1.14 \\
\hline
\end{tabular}

CUADRO 7

Lambdas. Planta ISI

Movilidad económica

\begin{tabular}{lccc} 
& Descendente & Sin movilidad & Ascendente \\
\hline Obreros directos & -0.25 & 0.54 & 0.29 \\
Mantenimiento & 0.25 & -0.54 & 0.29 \\
\hline
\end{tabular}

Podemos señalar que, en la empresa tradicional estudiada orientada al mercado interno, 1. a mayor antigüedad mejor el puesto ocupado; 2. a mayor calificación mejor el puesto ocupado; 3 . los trabajadores directos no tienen movilidad económica; 4. a mayor antigüedad los trabajadores de mantenimiento pasan de una movilidad económica descendente hacia una ascendente y, 5 . a mayor antigüedad los trabajadores de baja calificación pasan de la no movilidad a la movilidad económica descendente.

En síntesis, puede concluirse que la antigüedad es una variable que se asocia de manera importante con las otras analizadas. Por supuesto, 
el importante papel que juega la antigüedad en esta planta tradicional difícilmente se podría explicar, por un lado, si no existiera un sindicato fuerte y, por otro lado, una estructura rígida en la organización del trabajo basada en una multiplicidad de puestos parcelados, estrictamente definidos, y en la negociación empresa-sindicato por el control y ascenso en el trabajo.

\section{Planta Export}

La planta ubicada en el norte de México fue creada a mediados de los años ochenta bajo el concepto de la alta tecnología. Es una de las empresas de mayor tecnología en el país y en la escala Brigth el nivel de mecanización está caracterizado por automodificaciones de la acción de la máquina y por tanto es de alta automatización. Se dedica al ensamble de autos; trabaja tres modelos y exporta $\mathbf{1 0 0} \%$ de la producción hacia Estados Unidos y Canadá.

La evolución profesional se encuentra en el siguiente estado: producción automatizada y un creciente desarrollo de la mecanización y de la cadena automática. Disminuyen los obreros especializados y aumentan los calificados. El nivel técnico en el trabajo es más elevado que en la empresa orientada al mercado interno (ISI), las tolerancias son más estrictas y las instrucciones de las tarjetas de trabajo son más precisas. Se trata de determinar la mejor manera de controlar las contingencias para lograr la precisión (calidad) y productividad deseada. Obreros de máquinasherramientas y de sistemas computarizados. La producción se desarrolla en medio de una filosofía participativa, con trabajo flexible y se sustenta en los equipos de trabajo.

La planta tiene cerca de $\mathbf{1 0 0}$ robots del tipo point to point y cuenta con máquinas transfer. Los departamentos de estampado, soldadura y pintura están totalmente automatizados, y el sistema de inventarios justo a tiempo es llevado por computadoras. La organización del trabajo sigue un principio de sistema socio-técnico el cual apunta al desarrollo de la capacidad de iniciativa y a la toma de decisiones desde el trabajador y desde la unidad básica de producción conformada por los grupos de trabajo. Mantiene también un sistema de pago individual basado en las habilidades y conocimientos adquiridos por los trabajadores a partir de la rotación de las tareas de producción (polivalencia) y del cumplimiento de los programas individuales de capacitación. Con estas innovaciones se pretende el desplazamiento del "escalafón ciego" basado en la promoción del operario por su antigüedad y de manera secundaria por su capacitación.

Los puestos de trabajo no están definidos por ningún manual y de hecho sólo existe el puesto de "técnico universal"; la actividad de los trabajadores tiende a la polivalencia absoluta. Cada obrero debe estar habilitado para desempeñar diferentes puestos con distintos grados de dificul- 
tad y responsabilidad. El objetivo de la empresa es que cualquier obrero sea capaz de desempeñar todas las actividades del departamento, en un primer momento, y en toda la planta, en un segundo. La única cuota de producción es el número de autos que deben ser ensamblados diariamente. Su estructura de organización es flexible y funciona a través de los equipos de trabajo y los círculos de calidad. Todas las diferentes jerarquías de la empresa participan en el trabajo colectivo en forma intensa. Se trata de una revolución en el trabajo acompañada de una filosofía de la producción con conceptos flexibles y de involucramiento del trabajador con los intereses de la propia empresa. Aunque pertenece al mismo sindicato que la planta ISI, éste está lidereado por gente joven sin experiencia sindical anterior, y todo indica que la capacidad de decidir en el proceso de trabajo es menor que en la otra planta. Ejemplo de esto es que el contrato colectivo que rige fue diseñado por la empresa y el sindicato nacional y no tuvo la gente de esta planta ninguna oportunidad de participar en su elaboración. El resultado fue un contrato con pocas cláusulas favorables a los trabajadores y desligado de las decisiones en la producción y en el proceso de trabajo.

Los trabajadores son en su mayoría del estado donde se ubica la planta. Tienen en promedio 24 años de edad y una escasa experiencia laboral anterior. El nivel de escolaridad, en general, es de preparatoria completa o estudios técnicos terminados.

Los resultados del padrón de trabajadores sindicalizados fueron los siguientes: la planta tiene 17 puestos de trabajo distribuidos en 18 departamentos de producción. Las frecuencias fueron:

CUADRO 8

Características de la planta Export

\begin{tabular}{lrr}
\multicolumn{1}{c}{ Variables } & Frecuencias & Porcentaje \\
\hline Puesto & & \\
Obreros directos & 537 & 80.4 \\
Obreros de mantenimiento y control de calidad & 7 & 1.0 \\
Resto & 124 & 18.6 \\
Antigüedad & & \\
Baja (0-1 años) & 524 & 78.6 \\
Alta (1-3 años) & 143 & 21.4 \\
Calificación & & \\
Baja & 648 & 97.0 \\
Alta & 20 & 3.0 \\
Movilidad salarial & & \\
Descendente & 521 & 78.0 \\
Sin movilidad & 134 & 20.1 \\
Ascendente & 13 & 1.9 \\
\hline
\end{tabular}


Los datos sobre puesto de trabajo demuestran que la estructura ocupacional en esta planta está fundamentada en los obreros directos. Lo que indica empíricamente que la tesis sobre la tendencia a la desaparición de la tradicional dicotomía entre el trabajo directo y el de mantenimiento resulta válida estadísticamente para esta empresa automatizada y con nuevos conceptos de organización en el trabajo. Este fenómeno se puede explicar a partir de la estrategia central de las empresas modernas consistente en que el operario directo incorpore las funciones que antes venían haciendo los departamentos de mantenimiento y control de calidad.

La mayoría de los trabajadores tienen baja antigüedad (explicable fundamentalmente por lo reciente del establecimiento de la planta) y baja calificación. Resulta interesante observar cómo, al comparar las dos plantas, en la tradicional existen más trabajadores calificados que en la moderna, y en esta última, según entrevistas, el nivel de escolaridad promedio es mayor. ${ }^{11}$ Esto parecería apoyar la tesis de la descalificación de Brigth y Braverman, en dos sentidos: por un lado, indicaría que a mayor nivel de mecanización menor nivel de calificación, y por otro lado, que a mayor automatización mayor uso de trabajadores con niveles más altos de escolaridad. Sin embargo, esta tesis no se cumple debido a que estos autores no consideran que la calificación que están midiendo depende en gran parte de la clasificación que hace la empresa de los trabajadores con base en la antigüedad. Mientras que la antigüedad no es un indicador importante en Bright y Braverman y por tanto no se analiza, en este estudio resultó significativa su interacción en la planta considerada. Tampoco son analizadas por aquellos autores las políticas de movilidad en el trabajo, que difieren sustancialmente, en este estudio, entre las dos plantas ("escalafón ciego versus escalafón participativo"). Así, mientras aquellos autores analizan la calificación como un atributo de trabajo, dejan de lado que también representa un momento salarial. Los hallazgos presentados indican, por el contrario, que las categorías de calificación están relacionadas con políticas laborales y están definidas por las negociaciones empresasindicato.

Sorprenden también los datos sobre el número de trabajadores que perciben - según nómina- menos de lo que les corresponde por tabula-

11 Más que tratarse de trabajadores con un grado mayor o menor de calificación, de la clasificación que la empresa hace de sus trabajadores; es deciir, el mayor control de la empresa basado en una disminución de la capacidad de negociación sindical permite que los trabajadores empiecen con nivel 1 de categoría sin importar la calificación adquirida. Hay, por ejemplo. excelentes soldadores con conocimientos de neumática, hidráulica y estadística. Así, lo que estadísticamente aparece como menor calificación en la planta del norte, y que contradeciría los planteamientos teóricos expuestos a lo largo del documento, en realidad no es más que un mayor control empresarial en las decisiones de la calificación. El promedio de escolaridad en la planta del norte fue de 11 años contra 6 en la planta isı. (Datos obtenidos de entrevistas con líderes sindicales.) 
dor, lo que indica la menor capacidad de negociación del sindicato de esta planta, en comparación con la analizada anteriormente.

La hipótesis a contrastar en esta planta es que en el estado de evolución profesional de los operarios automatizados la calificación se asocia con el puesto de trabajo ocupado y la movilidad salarial. Para ser cautos en la explicación de la asociación entre la calificación y las otras variables y debido a que resultó una gran concentración de trabajadores en puestos directos $(\mathbf{8 0 . 4 \% )}$ y con movilidad descendente $(\mathbf{7 0 . 8 \%})$, conviene construir una proposición derivada indirectamente: en empresas automotrices automatizadas basadas en la organización flexible en el trabajo y orientadas hacia la exportación, no hay relación entre calificación y antigüedad.

Esta hipótesis se conecta teóricamente con la polivalencia en el trabajo que es buscada por las gerencias en plantas automatizadas. El aprendizaje y la movilidad en puestos y salarios no depende ahora fundamentalmente de la antigüedad, sino de la calificación. La calificación deja de ser un momento de la negociación colectiva para ser, una vez más, extraída de los trabajadores y pasar al control empresarial.

Se diseñó un modelo que relacionaba antigüedad, puesto y movilidad, por un lado, y la calificación con cada una de las variables, por el otro. Aunque tuvo buen ajuste, se decidió aceptar el modelo final generado, pues su ajuste fue mejor, refleja más claramente la estructura de los datos y permite contrastar más directamente la hipótesis.

Los resultados del "modelo loglineal final generado" fueron los siguientes: la calificación está relacionada con el puesto y con la movilidad salarial (tal y como lo propone la hipótesis). Pero la antigüedad también está relacionada con el puesto y con la movilidad salarial, es decir, sí existe un escalafón por antigüedad dentro de esta empresa (esto se explica porque cada año - según señala el contrato colectivo- pueden ascender los trabajadores a una sola categoría). Resalta el hecho de que los datos no arrojaron ninguna interacción entre calificación y antigüedad. El modelo generado con mejor ajuste y estructura más sencilla, queda expresado en la siguiente forma:

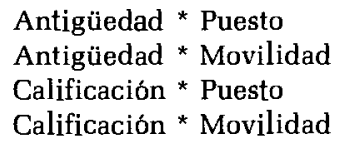

Este modelo relaciona antigüedad, puesto y movilidad, por un lado, y calificación, puesto y movilidad, por el otro. Cabe reiterar que el análisis no arrojó ninguna relación significativa entre calificación y antigüedad.

Los datos señalan una estructura compuesta por cuatro interacciones de dos variables cada una. Como puede apreciarse en los siguientes cuadros, los obreros directos son los que tienen baja antigüedad y los obreros 
de mantenimiento la tienen alta. Consistentemente, los obreros directos tienen baja calificación y los de mantenimiento alta.

CUADRO 9

Lambdas. Planta Export

\begin{tabular}{lcc}
\hline & \multicolumn{2}{c}{ Puesto } \\
\cline { 2 - 3 } Antigüedad & $\begin{array}{c}\text { Obreros } \\
\text { directos }\end{array}$ & $\begin{array}{c}\text { Obreros } \\
\text { mantenimiento }\end{array}$ \\
\hline Alta & -0.35 & 0.35 \\
Baja & 0.35 & -0.35 \\
\hline
\end{tabular}

CUADRO 10

Lambdas. Planta Export

\begin{tabular}{lcc}
\hline & \multicolumn{2}{c}{ Puesto } \\
\cline { 2 - 3 } Calificación & Obreros & $\begin{array}{c}\text { Obreros } \\
\text { mantenimiento }\end{array}$ \\
\hline Alta & directos & 0.99 \\
Baja & -0.99 & -0.99 \\
\hline
\end{tabular}

En lo que respecta a la movilidad económica, ésta es ascendente para los trabajadores que tienen alta antigüedad; y sin movilidad y descendente para los que tienen baja antigüedad. El comportamiento es similar para los trabajadores con alta y baja calificación, respectivamente, como puede observarse en los cuadros 11 y 12 .

CUADRO 11

Lambdas. Planta Export

\begin{tabular}{lccc}
\hline & \multicolumn{3}{c}{ Movilidad económica } \\
\cline { 2 - 4 } Antigüedad & Descendente & Sin movilidad & Ascendente \\
\hline Alta & -0.07 & -0.04 & 0.11 \\
Baja & 0.07 & 0.04 & -0.11 \\
\hline
\end{tabular}


CUADRO 12

Lambdas. Planta Export

\begin{tabular}{lccc}
\hline & \multicolumn{3}{c}{ Movilidad económica } \\
\cline { 2 - 4 } Calificación & Descendente & Sin movilidad & Ascendente \\
\hline Alta & -0.74 & -0.19 & 0.93 \\
Baja & 0.74 & 0.19 & -0.93 \\
\hline
\end{tabular}

Las interacciones demuestran que existe una política más racional en la planta moderna Export, donde a mayor antigüedad se avanza en el escalafón de puestos, por un lado, y también en la movilidad económica, por el otro. Algo similar sucede con la calificación. Los que tienen baja calificación ocupan puestos más bajos y tienen, básicamente, una movilidad descendente; conforme avanzan en la calificación, tiene puestos más altos y su movilidad es ascendente. De esta manera, la calificación determina el puesto de trabajo que se ocupa, por un lado, y la movilidad salarial, por el otro.

Al no estar relacionados la calificación y la antigüedad, la política de ascenso en esta empresa no se basa en la tradicional negociación empresa-sindicato, donde los puestos vacantes van siendo ocupados según la antigüedad ("al trabajador más antiguo le corresponde el ascenso"). Ahora la variable calificación tiene una mayor importancia, de tal forma que ésta es la que mejor explica los movimientos dentro de la empresa, $\mathrm{y}$ después la variable antigüedad.

Existe una mayor racionalidad en la administración del trabajo en la planta Export que en la planta ISI, producto de un rompimiento en el nivel de negociación alcanzado entre empresa y sindicato. Esta política más racional va acompañada de una menor capacidad colectiva de decisión de los trabajadores.

\section{Consideraciones finales}

La década de los ochenta se ha caracterizado por cambios sustanciales dentro de las empresas, particularmente en la industria automotriz. El cambio más visible es el del domicilio de la industria del auto. Mientras que el área metropolitana de la ciudad de México y los estados aledaños configuraron la región central de la producción automotriz, ahora son los municipios fronterizos y no fronterizos de los estados del norte de México el eje de producción del modelo exportador. Dicho cambio viene acompañado con transformaciones esenciales en la producción: productos, materiales, tecnología en maquinaria y equipo, y administración del trabajo. La comparación entre plantas tradicionales orientadas al mercado interno 
y plantas modernas orientadas a la exportación proporciona una buena comprensión de este proceso.

Aquí he analizado sólo el cambio en algunos factores relacionados con la calificación. Esto se circunscribe dentro de la polémica sobre las implicaciones en las transformaciones en la organización del trabajo y la tecnología, y su relación con el control sobre los trabajadores. Analizar la calificación en el trabajo es importante, pues es un concepto con un gran poder explicativo. Es un espacio donde se sintetizan los cambios técnicos, organizativos, laborales y de las estructuras de control. De ahí que sea de gran utilidad definirla como una situación de evolución profesional.

Los resultados del trabajo reflejan, en primer lugar, una estructura organizativa distinta en lo referente a departamentos de producción, puestos de trabajo y políticas de movilidad en la planta automatizada de creación reciente y en el complejo industrial establecido a principios de los años sesenta. Hay menos departamentos y puestos de trabajo en la planta Export que en la planta ISI. Y en segundo lugar señalan que en la planta tradicional el puesto que se va ocupando depende de la calificación y de la antigüedad. Sin embargo, la variable antigüedad tiene una mayor importancia, ya que significa una movilidad económica ascendente para los trabajadores de alta calificación y una movilidad económica descendente para los trabajadores de baja calificación. Esto es, si los trabajadores siguen la carrera profesional basada en la antigüedad, alcanzan mejores salarios y si no continúan la carrera profesional, su salario tiende a bajar relativamente. De tal forma que la movilidad ocupacional (movilidad en puestos y en salario) dentro del complejo está determinada básicamente por una política de negociación entre el sindicato y la empresa. El escalafón que promueve a los trabajadores según su antigüedad, y no necesariamente según su capacitación y calificación, es parte central de una política laboral que expresa relaciones más equilibradas de poder entre empresa y trabajadores con intermediación sindical.

Esta política laboral ha sido sustituida por la empresa, en la planta automatizada, por otra basada en la promoción del trabajador según capacitación, donde la antigüedad no tiene tanta importancia como la calificación. Resulta que a mayor calificación mejor el puesto que se ocupa, por un lado, y mayor la movilidad económica ascendente, por el otro. Otro hallazgo es que la antigüedad y la calificación no están relacionadas. El criterio de eficiencia (alta productividad, alta calidad y trabajo flexible) cobra en esta planta una gran relevancia.

Mi interpretación de este cambio en la política laboral concuerda con la de aquellos que consideran que, con la automatización en la producción y con los nuevos conceptos de organización, a la par que se incrementan los trabajos calificados, las responsabilidades y la flexibilidad, se centraliza el control sobre los trabajadores. Pero este control poco tiene que ver con la calificación per se, entendida como oficio. El trabajador 
no está perdiendo capacidad de decisión sobre la forma de trabajo con estas transformaciones; lo que está perdiendo, según señalan los resultados de los modelos, es un momento en la negociación de las políticas de trabajo. Si con el fordismo-tay lorismo le fue extraído al trabajador su conocimiento por la ciencia de la administración y fue entonces dirigido hacia las máquinas-herramientas, con la flexibilidad le están reduciendo los espacios de negociación laboral, al pasar del momento de negociación colectiva hacia la centralización del control por la empresa.

El cambio hacia la polivalencia en el trabajo implica, por tanto, la desaparición de puestos de mantenimiento y control de calidad, y la no correpondencia entre calificación y antigüedad. Visto así, la capacidad de negociación de los trabajadores y del sindicato disminuye en este sentido en plantas automatizadas, lo que se refleja en estructuras menos segmentadas de calificación y políticas distintas de ascenso.

\section{Bibliografía}

Arteaga, A. y Jorge Carrillo, "Automóvil, hacia la flexibilidad productiva", en El Cotidiano, UAM, núm. 21, enero-febrero de 1988, pp. 79-88.

Auguren, S. y F. Edgren, Neue Wege der Produktions und Fabrikplanung, Erfahrungen aus Schweden, Rationalisierungs-Kuratorium der Deutschen Wirtschat, RKW Nr. 855, 1986.

Babbage, Charles, On the economy of machinery and manufactures, 1832 (reeditada en Nueva York en 1963).

Barnet, Richard y R. Muller, Global Reach, Ed. Grijalbo, México, 1974.

Boyer, Robert, La flexibilidad del trabajo en Europa, Ministerio de Trabajo y Seguridad Social, Madrid, 1986.

Braverman, Harry, Trabajo y capital monopolista, Ed. Nuestro Tiempo, México, 1974.

Bright, J., "Automation and Management", en Harvard Business Review, Boston, julio-agosto de 1958.

Burawoy, Michael, "Between the labor process and the state: The changing face of factory regimes under advanced capitalism", en American Sociological Review, vol. 48, octubre de 1983, pp. 587-605.

Carrillo, Jorge, "Transformaciones en la industria maquiladora de exportación: ¿una nueva fase?", en Cuadernos Semestrales núm. 20, 20. semestre, cIDE, México, 1986, pp. 165-194.

Carrillo, Jorge y Patricia García, "Etapas industriales y conflictos laborales: Ia industria automotriz en Mexico", en Estudios Sociológicoss, vol. 5, núm. 14, mayoagosto de 1987.

Coriat, Benjamín, Ensayo sobre el taylorismo, el fordismo y la producción masiva, Siglo XXI, México, 1981.

De la Garza, Enrique, Seminario sobre procesos de trabajo y clase obrera, curso de actualización, México, UAM-I, abril-mayo de 1984. , "La investigación sobre la base obrera en México un balance preliminar", en Nueva Antropología, México, vol. VIII, núm. 29, abril, 1986, pp. 85-106.

Dhose, Knuth, et al., From fordism to toyostism. The social organization of the labour process in the japanese automovile industry, IIVG pre 84.218, Berlin, International Institute for Comparative Social Research \& Labor Policy, abril, 1984 . 
Dhose, Knuth, Ulrich Jurgens y Thomas Malsch, "Fertigungsnahe Selbstregulierung oder zentrale Kontrolle Konzernstrategien im Restrkturierungsproze \& der Automobilindustrie" en Frieder Naschold (compilador) Arbeit und Politik, Campus Verlag, Frankfurt/Nueva York, 1987.

Diebold, J., Automation and Automatic Equipment New, marzo de 1958.

Di Telia, Torcuato, La división del trabajo y el concepto marxista de clase social, Instituto Torcuato Di Telia, Buenos Aires, Trabajo Interno núm. 15, 1973.

Durkheim, Emile, La división del trabajo social, México, 1893 (reimpreso por UNAM sin fecha).

Frobel, F., J. Heinrich y O. Kreye, La nueva división internacional del trabajo, Siglo XXI, México, 1981.

Frost y Sullivan, "Predicción de un análisis de mèrcado", en Northern California Electronic News, enero 7 de 1980.

García, Brígida, Desarrollo económico y absorción de fuerza de trabajo en México:1950-1980, tesis de doctorado en sociología, UNAM, México, marzo de 1987.

Howard, Robert, "Cambios tecnológicos en telecomunicaciones" en Información obrera, núm. 1, México, verano, 1982, pp. 125-150.

Kern, H. y M. Schumann, Das Ende der Arbeitssssteiiung? Rationalisierung in der industriellen Produktion, Munich, 1984.

Lettieri, A., "Notas sobre las calificaciones, la escuela y los horarios de trabajo", en Cuadernos de Pasado y Presente, núm. 32, 1974, (4a. ed. 1980), pp. 133-150.

Malsch, Thomas, et al., Industrial robots in the automobile industry. A leap towards automated fordism, IIVG dp 84-222, Berlin, International Institute for Comparative Social Research \& Labor Policy, mayo de 1984.

Mertens, L. y C. Palomares, "El surgimiento de un nuevo tipo de trabajador en la industria de alta tecnología. El caso de la electrónica", en Esthela Gutiérrez, Restructuracion productiva y clase obrera, Siglo XXI, México, 1987.

Moritani, Masonori, Japanese technology. Getting the best for the least, Tokio, The Simul Press, 1985.

Naville, Pierre, "'División del trabajo y distribución de las tareas"', en G. Friedmann y P. Naville Tratado de Sociología del Trabajo I, FCE, México, 1963 (3a. reimpresión 1985).

Piore, M. y Ch. F. Sabel, The second industrial divide. Possibilities for prosperity, Basic Books, Nueva York, 1984.

Roxborough, Ian, Unions and politics in Mexico: the case of the automobile industry, Cambridge University Press, Latin American Studies, 1985.

Shaiken, Harley, Work transformed. Automation and labor in the computerage, Lexington, Lexington Books, 1986.

Shaiken, Harley y S. Herzenberg, Automation and global production, Monograph Series, 26, Center for US-Mexican Studies, University of California, San Diego, 1987.

Secretaría de Programación y Presupuesto, Estadísticas de la industria maquiladora de exportacion, México, SPP-INEGI (varios años).

Taylor, F.W., The Principies of Scientific Management, Mc Graw Hill, Nueva York, 1911.

Thompson, Paul, The nature of work: An introduction to debates on the labour Process, The McMillan Press, Londres, 1983.

Touraine, Alain, "La organización profesional de la empresa" en G.Freidman y P. Naville, Tratado de sociología del trabajo I, FCE, México, 1963 (3a. reimpresión, 1985), pp. 384-425.

Womack, James, Prospects for the US-Mexican relationship in the auto sector, Massachusetts, Center for Technology, Policy and Industrial Development, Massachusetts Institute of Technology, 1986 (manuscrito) 
